\title{
Opportunistic Spectrum Access in Support of Ultra-Reliable and Low Latency Communications
}

\author{
Wenjuan Yu, Atta ul Quddus, Seiamak Vahid, and Rahim Tafazolli \\ 5G Innovation Centre, Institute of Communication Systems, \\ University of Surrey, Guildford, GU2 7XH, UK \\ Emails: \{w.yu, a.quddus, s.vahid, r.tafazolli\}@ surrey.ac.uk
}

\begin{abstract}
This paper addresses the problem of opportunistic spectrum access in support of mission-critical ultra-reliable and low latency communications (URLLC). Considering the ability of supporting short packet transmissions in URLLC scenarios, a new capacity metric in finite blocklength regime is introduced as the traditional performance metrics such as ergodic capacity and outage capacity are no longer applicable. We focus on an opportunistic spectrum access system in which the secondary user (SU) opportunistically occupies the frequency resources of the primary user (PU) and transmits reliable short packets to its destination. An achievable rate maximization problem is then formulated for the SU in supporting URLLC services, subject to a probabilistic received-power constraint at the $P U$ receiver and imperfect channel knowledge of the SU-PU link. To tackle this problem, an optimal power allocation policy is proposed. Closedform expressions are then derived for the maximum achievable rate in finite blocklength regime, the approximate transmission rate at high signal-to-noise ratios (SNRs) and the optimal average power. Numerical results validate the accuracy of the proposed closed-form expressions and further reveal the impact of channel estimation error, block error probability, finite blocklength and received-power constraint.

Index Terms-URLLC, spectrum sharing, finite blocklength,
\end{abstract} achievable coding rate, imperfect channel information.

\section{INTRODUCTION}

It is noted that $5 \mathrm{G}$ wireless communications will support three generic services, i.e., enhanced mobile broadband (eMBB), massive machine-type communications (mMTC), and ultra-reliable and low-latency communications (URLLC) [1]. Specifically, URLLC refers to the scenarios where data packets are transmitted at moderately low throughput but with very high reliability (e.g., 99.999\%) and stringent latency requirements (e.g., $1 \mathrm{~ms}$ ) [2]. Apparently, the provision of URLLC has greatly emphasized the stringent requirements of reliability and end-to-end latency. Hence, it is vital and challenging for us to design suitable protocols and adaptive techniques, in support of the explosive growth of URLLC services in $5 \mathrm{G}$ networks, such as vehicular communications, tactile Internet and virtual reality [3]-[5].

In the context of URLLC, the main challenge is the support of intermittent short packets, which are the typical form of the traffic generated and exchanged in mission-critical communications [4]. Traditional information-theoretic metrics, such as ergodic capacity and outage capacity, are all defined for sufficiently large packet lengths. However, for short packet length communications, the aforementioned traditional capacity metrics become inaccurate and a new metric definition of the maximum achievable rate is required that takes into account the finite packet length and finite error probability [6], [7]. In [6], the authors derived an approximate expression for the maximum achievable rate in finite blocklength regime for a given error probability, which can be considered as a suitable capacity metric for URLLC. Hence, throughout this paper, we expand on the achievable rate expressions from [6] to evaluate the effective throughput for transmitting short packets.

On the other hand, due to the spectrum scarcity issue in future communication networks, it is important to investigate the performance of opportunistic spectrum access protocols in supporting URLLC services. With sufficient studies in classical cognitive radio networks, the applications of opportunistic spectrum access and corresponding access protocols in supporting URLLC scenarios remain inadequate [8]-[10]. For example, resource allocation strategies were analyzed and investigated in [11]-[13], aiming to improve system performance metrics in URLLC scenarios, such as energy efficiency and total transmission rate. However, the above mentioned papers mainly focused on providing efficient resource allocation algorithms, rather than proposing the analytical closed-form expressions and investigating the impact of different system parameters.

In this paper, we focus on an opportunistic spectrum sharing system, in which the secondary user (SU) opportunistically re-uses the spectrum resources of the primary user (PU), without bringing disruption at the PU receiver. Specifically, we assume that the SU supports URLLC services and transmits reliable short packets to its destination. With a new capacity metric introduced, the mathematical properties of the maximum achievable rate in finite blocklength regime are investigated first. Then, the optimal power control policy is proposed, followed by the analytical closed-form expressions. We summarize the primary contributions as follows:

- For an opportunistic spectrum access system, an optimal power control policy is studied for the SU in supporting short packet transmissions for URLLC scenarios. Specifically, the monotonicity of the achievable average rate in finite blocklength regime is theoretically proved.

- Closed-form expressions/approximations are derived for the maximum achievable rate in finite blocklength regime, the approximate rate at high signal-to-noise ratios (SNRs) and the optimal average power. The accuracy of the proposed closed-form expressions is validated through 


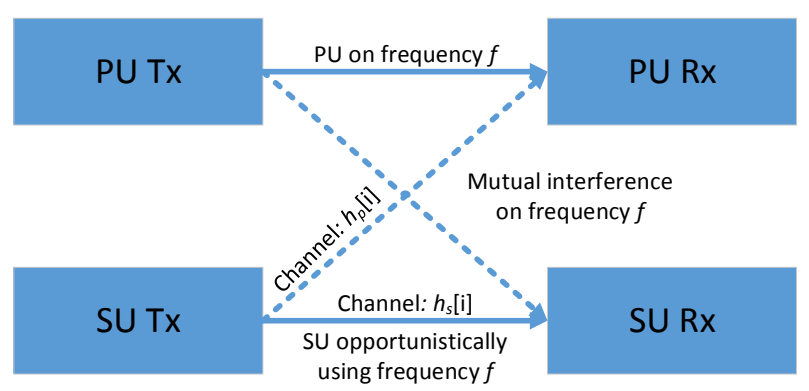

Fig. 1: System model.

Monte Carlo results.

- Numerical results further reveal that ergodic capacity achieved by adopting optimal power control serves as an upper bound for the achievable rate in finite blocklength regime. The gap between them can be reduced by carefully manipulating the blocklength, the error probability and the channel estimation error.

\section{SyStem ModeL}

The system model is illustrated in Fig. 1, in which the SU opportunistically occupies the PU's spectrum resources and supports reliable short packet transmissions in URLLC scenarios. The complex channel gain between the SU transmitter and the $\mathrm{SU}$ receiver is represented by $h_{s}[i]$, while $h_{p}[i]$ denotes the channel gain between the SU transmitter and the PU receiver. Block-fading channel models are considered [14], i.e., $h_{s}[i]$ and $h_{p}[i]$ stay fixed for the $i^{\text {th }}$ coherence interval and then independently change for the following interval. Further, it is assumed that $h_{p}[i]$ and $h_{s}[i]$ are independent and identically distributed (i.i.d.) zero mean circularly symmetric complex Gaussian (ZMCSCG) random variables, i.e., $\mathcal{C N}(0,1)$. Specifically, the SU transmitter and receiver are assumed to have perfect channel state information (CSI) of $h_{s}[i]$, but only have imperfect channel knowledge of $h_{p}[i]$ for the SU-PU link, which can be obtained through blind estimation techniques [15]. Let the minimum mean square error (MMSE) estimation for the fading coefficient $h_{p}[i]$ be $\hat{h}_{p}[i]$. The estimation error is denoted by

$$
\breve{h}_{p}[i]=h_{p}[i]-\hat{h}_{p}[i],
$$

which follows ZMCSCG distribution independent of $h_{p}[i]$, i.e., $\mathcal{C N}\left(0, \sigma_{e}^{2}\right)$. The channel estimation $\hat{h}_{p}[i]$ is thus ZMCSCG distributed with variance $1-\sigma_{e}^{2}$. When $\sigma_{e}^{2}=0$, it indicates that perfect channel knowledge is available.

Channel coding is assumed to be performed in each coherence interval of $m$ symbols [14]. For a given block error probability $\epsilon, 0<\epsilon<1$, a finite blocklength $m$, and signalto-noise ratio (SNR) $\rho$, the achievable instantaneous rate (bits per channel use (bpcu) [16]) for the SU in the $i^{\text {th }}$ interval can be approximated as [6]

$$
r_{i} \approx \log _{2}\left(1+\rho\left|h_{s}[i]\right|^{2}\right)
$$

$$
-\sqrt{\frac{1}{m}\left(1-\frac{1}{\left(\rho\left|h_{s}[i]\right|^{2}+1\right)^{2}}\right)} Q^{-1}(\epsilon) \log _{2}(e),
$$

where the approximation is accurate for $m \geq 100$ [6]. $Q^{-1}(x)$ is the inverse of the Gaussian Q-function with $Q(x)=$ $\int_{x}^{\infty} \frac{1}{\sqrt{2 \pi}} e^{-t^{2} / 2} d t$

Note that for short packet transmissions, finite error probability is inevitable. By assuming a block error probability $\epsilon$, the instantaneous rate $r_{i}$ in (2) is achieved with an errorfree probability $1-\epsilon$ and it becomes zero when an erroneous reception occurs. When a decoding error occurs, the SU receiver employing a simple automatic repeat request (ARQ) mechanisam will send a negative acknowledgement (NACK) requesting the retransmission. Hence, the effective service rate (in bits per $m$ channel uses) can be given as follows:

$$
R_{i}=\left\{\begin{aligned}
0, \text { with prob. } \epsilon \\
m r_{i}, \text { with prob. } 1-\epsilon .
\end{aligned}\right.
$$

Then, the achievable normalized average rate for the SU with finite blocklength codes, in bpcu, can be expressed as

$$
\bar{R}=\mathbb{E}\left[R_{i}\right]=(1-\epsilon) \mathbb{E}\left[r_{i}\right] .
$$

\section{Throughput with Finite Blocklength Codes}

Since the SU only has imperfect CSI of the SU-PU link, hence it cannot guarantee that the interference caused at the PU will remain below the peak limit [10]. Therefore, in this paper, we assume that the interference caused by the SU due to spectrum sharing may exceed the peak limit, but only for a very small percentage of time. By adopting optimal power control over time such that the received-power at the PU receiver is probabilistically constrained [17], the maximum average rate for the SU with finite blocklength codes can be represented as ${ }^{1}$

$$
\begin{aligned}
& \bar{R}=\max _{P_{g_{s}, \hat{g}_{p}}}(1-\epsilon) \mathbb{E}\left[\log _{2}\left(1+\frac{P_{g_{s}, \hat{g}_{p}} g_{s}}{N_{0} B}\right)\right. \\
&-\sqrt{\left.\frac{1}{m}\left(1-\frac{1}{\left(1+\frac{P_{g_{s}, \hat{g}_{p}} g_{s}}{N_{0} B}\right)^{2}}\right)\right]} Q^{-1}(\epsilon) \log _{2}(e), \\
& \text { subject to: } \operatorname{Pr}\left\{P_{\left.g_{s}, \hat{g}_{p} g_{p} \geq P_{\text {peak }}\right\}} \leq P_{\text {out }},\right.
\end{aligned}
$$

where $P_{g_{s}, \hat{g}_{p}}$ is the adaptive transmit power depending on the channel condition of the SU-SU link and the channel estimation of the SU-PU link, with $g_{s}=\left|h_{s}\right|^{2}$ and $\hat{g}_{p}=\left|\hat{h}_{p}\right|^{2}$. Further, $\mathbb{E}[\cdot]$ indicates the expectation over the joint probability density function (PDF) of $g_{s}$ and $\hat{g}_{p}, P_{\text {peak }}$ is the peak received-power limit, $P_{\text {out }}$ is the outage probability limit of the received-power, $N_{0} B$ is the additive white Gaussian noise (AWGN) power with $B$ denoting the channel bandwidth and $N_{0}$ indicating the single-sided noise spectral density. Hence, (5b) guarantees that the probability of the received-power at

\footnotetext{
${ }^{1}$ Hereafter, the time index $i$ is omitted for simplicity.
} 
the PU exceeding the peak value will be constrained by a given outage parameter $P_{\text {out }}$. For example, when $P_{\text {out }}=0.001$, it means that the received-power constraint $P_{g_{s}, \hat{g}_{p}} g_{p} \leq P_{\text {peak }}$ can be guaranteed for at least $99.9 \%$ of time.

Before solving the above optimization problem, we first analyze the received-power constraint (5b).

$$
\begin{aligned}
& \operatorname{Pr}\left\{P_{g_{s}, \hat{g}_{p}}\left(g_{p}\right) \geq P_{\text {peak }}\right\} \\
& =\operatorname{Pr}\left\{P_{g_{s}, \hat{g}_{p}}\left(\hat{g}_{p}+\breve{g}_{p}\right) \geq P_{\text {peak }}\right\} \\
& =\operatorname{Pr}\left\{\breve{g}_{p} \geq \frac{P_{\text {peak }}}{P_{g_{s}, \hat{g}_{p}}}-\hat{g}_{p}\right\} \\
& =\int_{0}^{\infty} f\left(g_{s}\right) d g_{s} \int_{0}^{\infty} f\left(\hat{g}_{p}\right) d \hat{g}_{p} \int_{\frac{P_{\text {peak }}}{P_{g_{s}, \hat{g}_{p}}}-\hat{g}_{p}}^{\infty} f\left(\breve{g}_{p}\right) d \breve{g}_{p},
\end{aligned}
$$

where $f\left(g_{s}\right), f\left(\hat{g}_{p}\right), f\left(\breve{g}_{p}\right)$ are the PDFs of $g_{s}, \hat{g}_{p}$, and $\breve{g}_{p}$, respectively. By inserting $f\left(g_{s}\right)=e^{-g_{s}}, f\left(\hat{g}_{p}\right)=$ $\frac{1}{1-\sigma_{e}^{2}} e^{-\frac{\hat{g}_{p}}{1-\sigma_{e}^{2}}}$, and $f\left(\breve{g}_{p}\right)=\frac{1}{\sigma_{e}^{2}} e^{-\frac{\breve{g}_{p}}{\sigma_{e}^{2}}}$ into (6d), the received-power outage constraint $(5 \mathrm{~b})$ can be expressed as

$$
\int_{0}^{\infty} e^{-g_{s}} \int_{0}^{\infty} e^{-\frac{P_{\text {peak }}}{\sigma_{e}^{2} P_{g_{s}, \hat{g}_{p}}}+\frac{\hat{g}_{p}}{\sigma_{e}^{2}}} \frac{e^{-\frac{\hat{g}_{p}}{1-\sigma_{e}^{2}}}}{1-\sigma_{e}^{2}} d \hat{g}_{p} d g_{s} \leq P_{\text {out }}
$$

Note that obtaining the optimal power allocation policy which maximizes the achievable average rate, subject to the received-power constraint (7a) is difficult [10]. However, one sufficient instantaneous power constraint can be found whose satisfaction guarantees that the received-power constraint (7a) will be met at all times, which is [10]

$$
P_{g_{s}, \hat{g}_{p}} \leq \frac{P_{\text {peak }}}{\hat{g}_{p}-\sigma_{e}^{2} \ln P_{\text {out }}}
$$

After applying the sufficient instantaneous power constraint (8), the original rate maximization problem for the SU transmitting short packets in URLLC, i.e., (5a)-(5b), becomes

$$
\begin{aligned}
& \bar{R}=\max _{P_{g_{s}, \hat{g}_{p}}}(1-\epsilon) \mathbb{E}\left[\log _{2}\left(1+\frac{P_{g_{s}, \hat{g}_{p}} g_{s}}{N_{0} B}\right)\right. \\
& -\sqrt{\frac{1}{m}\left(1-\frac{1}{\left(1+\frac{P_{g_{s}, \hat{g}_{p} g_{s}} N_{0} B}{N_{0}}\right)}\right]} Q^{-1}(\epsilon) \log _{2}(e) \\
& \text { subject to: } P_{g_{s}, \hat{g}_{p}} \leq \frac{P_{\mathrm{peak}}}{\hat{g}_{p}-\sigma_{e}^{2} \ln P_{\mathrm{out}}} \text {. }
\end{aligned}
$$

We note that the main difficulty of solving the above optimization problem $(9 a)-(9 b)$ is that the objective function is non-convex and more complicated, compared to the traditional ergodic capacity. Hence, in order to optimally solve it, we start by analyzing the property of the objective function.

Theorem 1: For sufficiently large values of transmit power, the achievable average rate $\bar{R}$ monotonically increases with $P_{g_{s}, \hat{g}_{p}}$. Specifically, under some practical assumptions relevant to URLLC scenarios, i.e., $m \geq 100, \epsilon>10^{-6}$, the instantaneous rate $r_{i}$ for an AWGN channel monotonically increases with the transmit SNR $\rho_{0}$, for $\rho_{0} \geq-10 \mathrm{~dB}^{2}$.

Proof: See Appendix A.

Theorem 1 first proves that for the fading SU-SU channel, the achievable average rate $\bar{R}$ is a monotonically increasing function for sufficiently large power values. In order to obtain a more general conclusion, we further take an AWGN channel as an example, which indicates that under some reasonable assumptions on $m$ and $\epsilon$ [2], [18], the instantaneous rate $r_{i}$ can be proved to be monotonically increasing with the transmit SNR $\rho_{0}$, as long as $\rho_{0}$ is not extremely low [19]. In this context, we can note that for non-extremely low SNR values, the optimal power control policy which solves the maximization problem (9a)-(9b) is simply transmitting at the maximum instantaneous power limit, i.e., $P_{g_{s}, \hat{g}_{p}}=\frac{P_{\text {peak }}}{\hat{g}_{p}-\sigma_{e}^{2} \ln P_{\text {out }}}$.

By inserting the optimal power value into the objective function, we can get that the achievable rate $\bar{R}$ equals to

$$
(1-\epsilon) \mathbb{E}\left[\log _{2}\left(1+\frac{P_{\text {peak }} g_{s}}{N_{0} B\left(\hat{g}_{p}-\sigma_{e}^{2} \ln P_{\text {out }}\right)}\right)-\beta_{\mathrm{m}, \epsilon} \sqrt{V}\right],
$$

where $V=1-\frac{1}{\left(1+\frac{P_{\text {peak }} g_{s}}{N_{0} B\left(\hat{g}_{p}-\sigma_{e}^{2} \ln P_{\text {out }}\right)}\right)^{2}}$ and $\beta_{\mathrm{m}, \epsilon}=$ $\sqrt{\frac{1}{m}} Q^{-1}(\epsilon) \log _{2} e$. Furthermore, the optimal average power can be expressed as

$$
\bar{P}=\mathbb{E}\left[P_{g_{s}, \hat{g}_{p}}\right]=\mathbb{E}\left[\frac{P_{\text {peak }}}{\hat{g}_{p}-\sigma_{e}^{2} \ln P_{\text {out }}}\right] .
$$

Theorem 2: Considering imperfect CSI of the SU-PU link and the received-power constraint at the PU receiver, a closedform approximation for $\bar{R}$ is given in (11) (shown on next page), where $\eta_{1}=\frac{N_{0} B}{P_{\text {peak }}}, \eta_{2}=\frac{N_{0} B \sigma_{e}^{2} \ln P_{\text {out }}}{P_{\text {peak }}}, e_{m}(x)=$ $\sum_{k=0}^{m} \frac{x^{k}}{k !}$, and $E_{1}(\cdot)$ is the exponential integral function [20]. Further, the optimal average transmit power $\bar{P}$ for the SU is expressed in closed-form, given in (12) (shown on next page).

Proof: The proof for deriving the closed-form for $\bar{R}$ is provided in Appendix B, while the proof for deriving the closed-form for $\bar{P}$ is omitted here due to page limit, but it can be obtained by following similar steps as in [10].

At high SNRs, it is known that $\lim _{\rho \rightarrow \infty} V=1$ [6], [7]. In this case, the achievable rate for the SU in URLLC, denoted by $\hat{R}$, can be expressed as

$\hat{R}=(1-\epsilon)\left(\mathbb{E}\left[\log _{2}\left(1+\frac{P_{\mathrm{peak}} g_{s}}{N_{0} B\left(\hat{g}_{p}-\sigma_{e}^{2} \ln P_{\text {out }}\right)}\right)\right]-\beta_{\mathrm{m}, \epsilon}\right)$.

Lemma 1: At high SNRs, the closed-form expression for the achievable rate $\hat{R}$ is given in (13) (shown on next page) for the SU with imperfect CSI of the SU-PU link, under an received-power outage constraint at the PU receiver.

\footnotetext{
${ }^{2}$ Here, $\rho_{0}$ is the transmit SNR for an AWGN channel.
} 


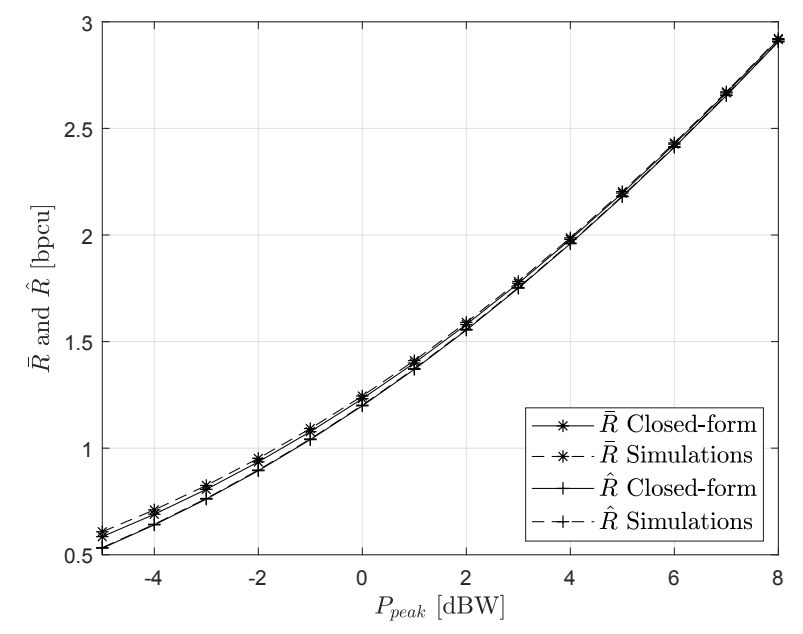

Fig. 2: The maximum achievable rates with finite blocklength codes, $\bar{R}$ and $\hat{R}$ at high SNRs, versus peak received-power limit $P_{\text {peak }}$.

Proof: Closed-form expression for $\hat{R}$ at high SNRs can be derived by following similar steps as in Appendix B.

\section{NumericAl RESUlts}

In this section, all the theorems and lemmas proposed in Section III will be numerically validated. The impact of blocklength $m$, error probability $\epsilon$, channel estimation error, outage parameter $P_{\text {out }}$ and peak received-power limit $P_{\text {peak }}$ will also be discussed and analyzed.

Firstly, in order to confirm the accuracy of the proposed closed-form expressions for the maximum achievable rates for the SU transmitting short packets, i.e., $\bar{R}$ and $\hat{R}$, we include Fig. 2 which shows the plots of $\bar{R}$ and $\hat{R}$ versus $P_{\text {peak }}$. Closedform expressions are plotted in solid lines, while the Monte Carlo simulations are plotted in dash lines. To plot this figure, it is assumed that the channel estimation error variance $\sigma_{e}^{2}=$ 0.001 , the outage parameter $P_{\text {out }}=0.001$, the block error probability $\epsilon=10^{-3}$, and the blocklength $m=500$ symbols. From Fig. 2, we can notice that the closed-form expression for $\hat{R}$ at high SNRs exactly matches with the Monte Carlo results. The closed-form approximation for $\bar{R}$ matches with numerical results when $P_{\text {peak }}$ is sufficiently large, e.g., $P_{\text {peak }} \geq 0 \mathrm{dBW}$, and has a negligible difference with Monte Carlo results when $P_{\text {peak }}$ is small, e.g., $P_{\text {peak }} \leq-2 \mathrm{dBW}$. This is because that we use an approximation step of $\sqrt{1-x} \approx 1-1 / 2 x$ to derive the closed-form approximation for $\bar{R}$, which results in slight imprecision due to the omitted remainder terms of Binomial series. Furthermore, Fig. 2 also shows that at high $P_{\text {peak }}$ values, the maximum achievable rate at high SNRs, i.e., $\hat{R}$, matches with $\bar{R}$, which confirms Lemma 1 .

To investigate the impact of the outage parameter $P_{\text {out }}$ and the received-power limit $P_{\text {peak }}$ on the achievable rate performance for the SU in URLLC, we include Fig. 3 which plots the curves of $\bar{R}$ and $C$ versus the peak received-power limit $P_{\text {peak }}$, for various values of $P_{\text {out }}$. To plot this figure, it is

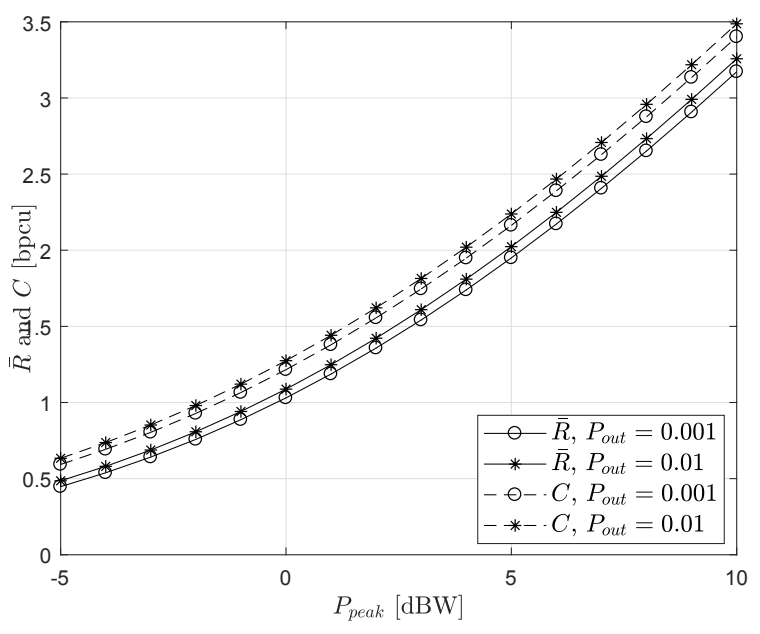

Fig. 3: The maximum achievable rate with finite blocklength codes $\bar{R}$ and ergodic capacity $C$ versus peak received-power limit $P_{\text {peak }}$, for various values of $P_{\text {out }}$.

assumed that the channel estimation error variance $\sigma_{e}^{2}=0.01$, the error probability $\epsilon=10^{-4}$, and the blocklength $m=500$ symbols. Firstly, one can notice from Fig. 3 that the ergodic capacity $C$ always serves as an upper bound for the achievable rate $\bar{R}$ in finite blocklength regime. This is because that the instantaneous rate using finite blocklength codes, i.e., $r_{i}$, can be seen as an expression derived by incurring a penalty on the traditional throughput, in which the penalty depends on the desired error probability and a given blocklength. Secondly, for a fixed value of $P_{\text {peak }}$, Fig. 3 indicates that when $P_{\text {out }}$ becomes larger, the values of $\bar{R}$ and $C$ becomes larger. This is because with a larger value of $P_{\text {out }}$, the received power at the PU has a higher probability of exceeding the peak limit $P_{\text {peak }}$, which leads to higher instantaneous transmit power and consequently, the maximum transmission rate becomes larger. However, in order to guarantee that PU is not disturbed, the SU prefers to keep the outage parameter $P_{\text {out }}$ as small as possible. From Fig. 3, we can notice that for a fixed $P_{\text {peak }}$ value, when $P_{\text {out }}$ is reduced from 0.01 to 0.001 , it only results in very negligible compromise on the maximum achievable rate $\bar{R}$.

To investigate the impact of the block error probability and the channel estimation error on the achievable rate performance for the SU in URLLC, Fig. 4 is included which plots the curves of $\bar{R}$ and ergodic capacity $C$ versus the blocklength $m$, for various values of channel estimation error variance $\sigma_{e}^{2}$ and block error probability $\epsilon$. Note that this figure is plotted using Monte Carlo simulations, by assuming that $P_{\text {peak }}=0 \mathrm{dBW}$ and the outage parameter $P_{\text {out }}=0.01$. From this figure, one can first note that for a fixed parameter setting, e.g., $\sigma_{e}^{2}=0.01$ and $\epsilon=10^{-2}$, the maximum achievable rate $\bar{R}$ for the SU in finite blocklength regime monotonically increases with the blocklength $m$, while the ergodic capacity is independent of the blocklength. This is due to the reason that the penalty added on the instantaneous achievable rate with finite blocklength codes is proportional to $1 / \sqrt{m}$ [7]. 


$$
\begin{aligned}
& \bar{R} \approx(1-\epsilon)\left(\frac{P_{\text {peak }}}{\ln 2\left(P_{\text {peak }}-\left(1-\sigma_{e}^{2}\right) N_{0} B\right)}\left(-e^{-\frac{\sigma_{e}^{2} \ln P_{\text {out }}}{1-\sigma_{e}^{2}}} E_{1}\left(-\frac{\sigma_{e}^{2} \ln P_{\text {out }}}{1-\sigma_{e}^{2}}\right)+e^{-\eta_{2}} E_{1}\left(-\eta_{2}\right)\right)-\frac{\beta_{\mathrm{m}, \epsilon}}{1-\sigma_{e}^{2}}\left(1-\sigma_{e}^{2}\right.\right. \\
& -\frac{1}{2} \eta_{1}\left(1-\sigma_{e}^{2}\right)^{2}+\frac{1}{2} \eta_{2}\left(1-\sigma_{e}^{2}\right)-\frac{\frac{1}{\eta_{1}} e^{-\frac{\eta_{2}}{\left(1-\sigma_{e}^{2}\right) \eta_{1}}}}{\left(\frac{1}{\eta_{1}\left(1-\sigma_{e}^{2}\right)}-1\right)^{3}}\left(E_{1}\left(-\frac{\eta_{2}}{\eta_{1}\left(1-\sigma_{e}^{2}\right)}\right)-e_{2}\left(-\eta_{2}\left(\frac{1}{\eta_{1}\left(1-\sigma_{e}^{2}\right)}-1\right)\right)\right. \\
& \left.\left.\times e^{\eta_{2}\left(\frac{1}{\eta_{1}\left(1-\sigma_{e}^{2}\right)}-1\right)} E_{1}\left(-\eta_{2}\right)+e^{\frac{\eta_{2}}{\eta_{1}\left(1-\sigma_{e}^{2}\right)}} \sum_{m=1}^{2} \frac{1}{m} e_{m-1}\left(-\frac{\eta_{2}}{\eta_{1}\left(1-\sigma_{e}^{2}\right)}\right)\left(1+\frac{1}{\frac{1}{\eta_{1}\left(1-\sigma_{e}^{2}\right)}-1}\right)\right)\right) \\
& \bar{P}=\frac{P_{\text {peak }}}{1-\sigma_{e}^{2}} e^{-\frac{\sigma_{e}^{2} \ln P_{\text {out }}}{1-\sigma_{e}^{2}}} E_{1}\left(-\frac{\sigma_{e}^{2} \ln P_{\text {out }}}{1-\sigma_{e}^{2}}\right),
\end{aligned}
$$$$
\hat{R}=(1-\epsilon)\left(\frac{P_{\text {peak }}}{\ln 2\left(P_{\text {peak }}-\left(1-\sigma_{e}^{2}\right) N_{0} B\right)}\left(-e^{-\frac{\sigma_{e}^{2} \ln P_{\text {out }}}{1-\sigma_{e}^{2}}} E_{1}\left(-\frac{\sigma_{e}^{2} \ln P_{\mathrm{out}}}{1-\sigma_{e}^{2}}\right)+e^{-\eta_{2}} E_{1}\left(-\eta_{2}\right)\right)-\beta_{\mathrm{m}, \epsilon}\right) .
$$

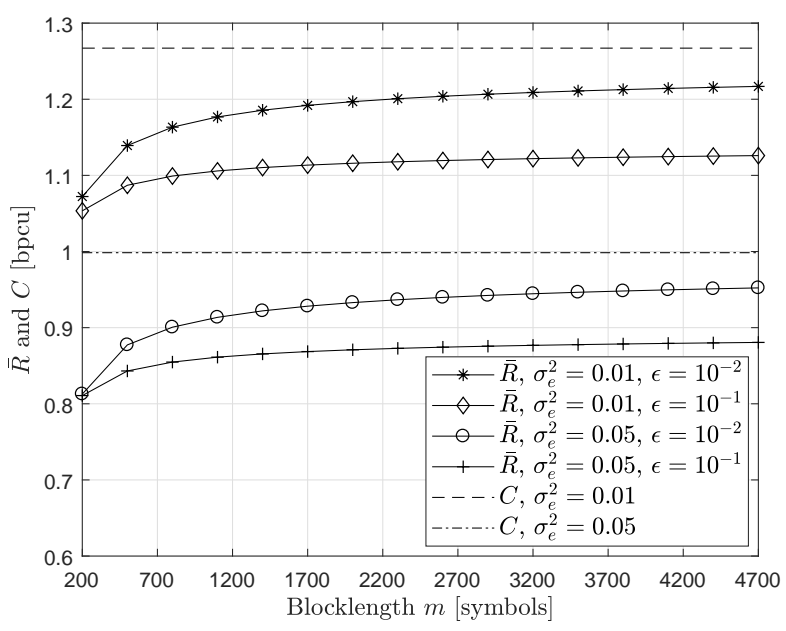

Fig. 4: The maximum achievable rate with finite blocklength codes $\bar{R}$ and ergodic capacity $C$ versus blocklength $m$, for various values of $\sigma_{e}^{2}$ and $\epsilon$.

Secondly, for a fixed setting of $m$ and $\epsilon$, when $\sigma_{e}^{2}$ becomes larger, i.e., changing from 0.01 to 0.05 , the achievable rate $\bar{R}$ for the SU becomes smaller. This indicates that the channel estimation error will degrade the achievable rate performance in finite blocklength regime. Thirdly, when the values of $m$ and $\sigma_{e}^{2}$ are fixed, Fig. 4 shows that the $\bar{R}$ value obtained with a smaller $\epsilon$, i.e., $\epsilon=10^{-2}$, is larger than the one obtained with $\epsilon=10^{-1}$.

Finally, from the optimal average transmit power expression, given in (10), we can note that the value of $\bar{P}$ for the SU is independent of $\epsilon$ and $m$, and only depends on the values of $\sigma_{e}^{2}$, $P_{\text {out }}$ and $P_{\text {peak }}$. Hence, Fig. 5 is plotted, in order to show the accuracy of the proposed closed-form expression for $\bar{P}$ and

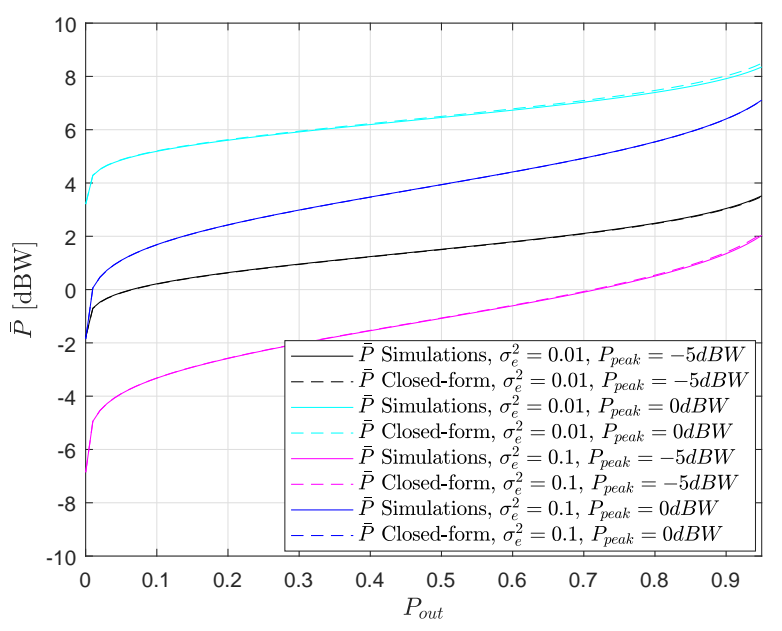

Fig. 5: The optimal average power versus outage parameter $P_{\text {out }}$, for various values of $\sigma_{e}^{2}$ and $P_{\text {peak }}$.

also to investigate the impact of $\sigma_{e}^{2}, P_{\text {out }}$ and $P_{\text {peak }}$ on the optimal average power value. To plot this figure, it is assumed that $\epsilon=10^{-4}$ and $m=1000$ symbols. Firstly, from this figure, we can note that the proposed closed-form expression for $\bar{P}$ (in dash lines) confirms with the Monte Carlo simulations (in solid lines), which guarantees the accuracy. Secondly, we can note that $\bar{P}$ monotonically increases with $P_{\text {out }}$, for each fixed setting of $\sigma_{e}^{2}$ and $P_{\text {peak }}$. Intuitively, a larger $P_{\text {out }}$ value means that the instantaneous received-power at the PU receiver can exceed the peak limit $P_{\text {peak }}$ with a higher probability, which results in a higher average transmit power for the SU. Furthermore, Fig. 5 shows that $\bar{P}$ is larger for a smaller $\sigma_{e}^{2}$ value and a larger $P_{\text {peak }}$ value. This phenomenon can be easily 
justified by analyzing the initial expression of (10), which is omitted here.

\section{CONCLUSIONS}

This paper investigated the opportunistic spectrum access design in supporting URLLC services, in which the SU opportunistically re-uses the frequency resources of the PU and send reliable short packets to its destination, without bringing disruption at the PU receiver. A new achievable rate metric was adopted in this paper, which is more suitable for representing URLLC services. In order to maximize the achievable data rate for the SU, an optimal power control policy was proposed, subject to a probabilistic received-power constraint and imperfect channel information of the SU-PU link. Numerical results confirmed the accuracy of the proposed closed-form expressions and further indicated that, the ergodic capacity always serves as an upper bound for the achievable rate in finite blocklength regime, and the gap between them can be reduced by carefully manipulating the values of blocklength, error probability and also the channel estimation error.

\section{AppendiX A: Proof of Theorem 1}

Recall that the achievable average rate $\bar{R}$ for the SU using finite blocklength codes is $\bar{R}=(1-\epsilon) \mathbb{E}\left[r_{i}\right]$. For simplicity, in the following $\mathbb{E}\left[r_{i}\right]$ is denoted by $\bar{r}$, which equals to

$$
\mathbb{E}\left[\log _{2}\left(1+\rho g_{s}\right)-\sqrt{\frac{1}{m}\left(1-\frac{1}{\left(\rho g_{s}+1\right)^{2}}\right)} Q^{-1}(\epsilon) \log _{2} e\right] .
$$

Here, $\rho$ is the transmit SNR for the SU-SU fading channel, i.e., $\rho=\frac{P_{g_{s}, \hat{g}_{p}}}{N_{0} B}$. When $\rho$ becomes sufficiently large, one can notice that

$$
\begin{aligned}
\lim _{\rho \rightarrow \infty} \mathbb{E} & {\left[\sqrt{\frac{1}{m}\left(1-\frac{1}{\left(\rho g_{s}+1\right)^{2}}\right)} Q^{-1}(\epsilon) \log _{2} e\right] } \\
& =\sqrt{\frac{1}{m}} Q^{-1}(\epsilon) \log _{2} e .
\end{aligned}
$$

Then, the achievable average rate $\bar{R}$ is dominated by the logarithm term, i.e., $\mathbb{E}\left[\log _{2}\left(1+\rho g_{s}\right)\right]$, which is known to be a monotonically increasing function with $\rho$. Since the transmit power $P_{g_{s}, \hat{g}_{p}}$ is basically the transmit SNR $\rho$ multiplied by the constant noise power, hence we can conclude that for sufficiently large transmit power values, the achievable average rate $\bar{R}$ monotonically increases with $P_{g_{s}, \hat{g}_{p}}$.

Then, we note that for an AWN channel, $r_{i}$ is given as

$$
\log _{2}\left(1+\rho_{0}\right)-\sqrt{\frac{1}{m}\left(1-\frac{1}{\left(1+\rho_{0}\right)^{2}}\right)} Q^{-1}(\epsilon) \log _{2} e,
$$

where $\rho_{0}$ is the transmit SNR for an AWGN channel. By taking the first derivative, we can get that $\frac{\partial r_{i}}{\partial \rho_{0}}$ equals to

$$
\frac{\log _{2} e}{1+\rho_{0}}\left(1-\sqrt{\frac{1}{m}} Q^{-1}(\epsilon)\left(1-\frac{1}{\left(1+\rho_{0}\right)^{2}}\right)^{-\frac{1}{2}}\left(1+\rho_{0}\right)^{-2}\right) .
$$

For URLLC scenarios, it is reasonable to assume $m \geq$ 100 and $\epsilon \geq 10^{-6}$ [2]. Hence, we can get that $\sqrt{\frac{1}{m}} \leq \sqrt{\frac{1}{100}}$ and $Q^{-1}(\epsilon) \leq Q^{-1}\left(10^{-6}\right)^{3}$. Further, since $\left(1-\frac{1}{\left(1+\rho_{0}\right)^{2}}\right)^{-\frac{1}{2}}\left(1+\rho_{0}\right)^{-2}$ monotonically decreases with $\rho_{0}$, therefore we can finally prove that under the reasonable assumptions of $m \geq 100$ and $\epsilon \geq 10^{-6}$, $\sqrt{\frac{1}{m}} Q^{-1}(\epsilon)\left(1-\frac{1}{\left(1+\rho_{0}\right)^{2}}\right)^{-\frac{1}{2}}\left(1+\rho_{0}\right)^{-2}<1$, as long as $\rho_{0}$ is not extremely low, i.e., $\rho_{0} \geq-10 \mathrm{~dB}$.

\section{Appendix B: Proof of Theorem 2}

Recall that $\bar{R}=(1-\epsilon) \bar{r}$, where $\bar{r}=C-\beta_{\mathrm{m}, \epsilon} \mathbb{E}[\sqrt{V}]$. Here, $C$ is ergodic capacity obtained at the instantaneous power limit, whose closed-form expression can be obtained by following similar steps in [10]. Here, we only provide the proof for deriving the closed-form for $\mathbb{E}[\sqrt{V}]$, yielding

$$
\begin{aligned}
& \int_{0}^{\infty} \frac{e^{-\frac{\hat{g}_{p}}{1-\sigma_{e}^{2}}}}{1-\sigma_{e}^{2}} \int_{0}^{\infty} \sqrt{1-\frac{1}{\left(1+\frac{P_{\text {peak }} g_{s}}{N_{0} B\left(\hat{g}_{p}-\sigma_{e}^{2} \ln P_{\text {out }}\right)}\right)^{2}}} \\
& \times e^{-g_{s}} d g_{s} d \hat{g}_{p} .
\end{aligned}
$$

Note that $\sqrt{1-x} \approx 1-\frac{1}{2} x$, for $|x|<1$. Then, by applying this approximation and one substitution step of $g_{s}=\frac{N_{0} B\left(\hat{g}_{p}-\sigma_{e}^{2} \ln P_{\text {out }}\right) x}{P_{\text {peak }}},(15)$ can be approximated as

$$
\begin{aligned}
& \mathbb{E}[\sqrt{V}] \approx \int_{0}^{\infty} \frac{e^{-\frac{\hat{g}_{p}}{1-\sigma_{e}^{2}}}}{1-\sigma_{e}^{2}}\left(1-\frac{1}{2} \frac{N_{0} B\left(\hat{g}_{p}-\sigma_{e}^{2} \ln P_{\text {out }}\right)}{P_{\text {peak }}}\right. \\
& \left.\times \int_{0}^{\infty}(1+x)^{-2} e^{-\frac{N_{0} B\left(\hat{g}_{p}-\sigma_{e}^{2} \ln P_{\text {out }}\right) x}{P_{\text {peak }}}} d x\right) d \hat{g}_{p} .
\end{aligned}
$$

According to (3.353.3) in [21], we note that

$$
\int_{0}^{\infty} \frac{e^{-p x}}{(a+x)^{2}} d x=p e^{a p} E_{i}(-a p)+\frac{1}{a}, p>0, a>0 .
$$

By applying (17), (16) can be expressed as

$$
\mathbb{E}[\sqrt{V}] \approx \frac{1}{1-\sigma_{e}^{2}}\left(1-\sigma_{e}^{2}-\frac{N_{0} B}{2 P_{\mathrm{peak}}}\left(1-\sigma_{e}^{2}\right)^{2}\right.
$$

${ }^{3}$ This is because $Q^{-1}(x)$ is a monotonically decreasing function with $x$. 


$$
\left.+\frac{N_{0} B \sigma_{e}^{2} \ln P_{\text {out }}}{2 P_{\text {peak }}}\left(1-\sigma_{e}^{2}\right)-A_{1}\right)
$$

where $A_{1}$ is given as

$A_{1}=-\frac{1}{2 \eta_{1}} e^{-\frac{\eta_{2}}{\left(1-\sigma_{e}^{2}\right) \eta_{1}}} \int_{-\eta_{2}}^{\infty} y^{2} e^{\left(1-\frac{1}{\eta_{1}\left(1-\sigma_{e}^{2}\right)}\right) y} E_{1}(y) d y$, where $\eta_{1}=\frac{N_{0} B}{P_{\text {peak }}}, \eta_{2}=\frac{N_{0} B \sigma_{e}^{2} \ln P_{\text {out }}}{P_{\text {peak }}}$. To find the closedform expression for $A_{1}$, we note that (4.2.16) in [22] provides

$$
\begin{aligned}
& \int x^{n} e^{-a x} E_{1}(b x) d x=\frac{n !}{a^{n+1}} E_{1}((a+b) x)-\frac{n !}{a^{n+1}} e_{n}(a x) \\
& \times e^{-a x} E_{1}(b x)+\frac{n !}{a^{n+1}} e^{-(a+b) x} \sum_{m=1}^{n} \frac{e_{m-1}((a+b) x)}{m\left(1+\frac{b}{a}\right)^{m}},
\end{aligned}
$$

where $e_{m}(x)=\sum_{k=0}^{m} \frac{x^{k}}{k !}$. By applying (19), $A_{1}$ can be expressed in closed-form and the closed-form approximation for $\mathbb{E}[\sqrt{V}]$ can then be obtained by inserting $A_{1}$ back into (18). Finally, the closed-form approximation for $\bar{R}$ can be derived by using $\bar{R}=(1-\epsilon)\left(C-\beta_{\mathrm{m}, \epsilon} \mathbb{E}[\sqrt{V}]\right)$ and is given in (11).

\section{ACKNOWLEDGEMENT}

This work was partly funded by grant N62909-17-1-2114 from the US Office of Naval Research Global. In addition, the authors would also like to acknowledge the support of the University of Surrey 5GIC (http://www.surrey.ac.uk/5gic) members for this work.

\section{REFERENCES}

[1] 3GPP, "Study on scenarios and requirements for next generation access technologies," 3GPP 38.913 Version 15, Tech. Rep., 2018.

[2] G. Durisi, T. Koch, and P. Popovski. (2015) Towards massive, ultrareliable, and low-latency wireless communication with short packets. [Online]. Available: arXiv:1504.06526

[3] W. Yu, L. Musavian, and Q. Ni, "Link-layer capacity of NOMA under statistical delay QoS guarantees," IEEE Trans. Commun., in press.

[4] M. Bennis, M. Debbah, and H. V. Poor. (2018) Ultra-reliable and low-latency wireless communication: Tail, risk and scale. [Online]. Available: arXiv:1801.01270

[5] W. Yu, L. Musavian, and Q. Ni, "Tradeoff analysis and joint optimization of link-layer energy efficiency and effective capacity toward green communications," IEEE Trans. Wireless Commun., vol. 15, no. 5, pp. 3339-3353, Jan. 2016.

[6] Y. Polyanskiy, H. V. Poor, and S. Verdu, "Channel coding rate in the finite blocklength regime," IEEE Trans. Inf. Theory, vol. 56, no. 5, pp. 2307-2359, May 2010.

[7] S. Schiessl, H. Al-Zubaidy, M. Skoglund, and J. Gross. (2016) Delay performance of wireless communications with imperfect CSI and finite length coding. [Online]. Available: arXiv:1608.08445

[8] Y. Liu, Z. Ding, M. Elkashlan, and J. Yuan, "Nonorthogonal multiple access in large-scale underlay cognitive radio networks," IEEE Trans. Veh. Technol., vol. 65, no. 12, pp. 10 152-10 157, Dec. 2016.

[9] L. Lv, Q. Ni, Z. Ding, and J. Chen, "Cooperative non-orthogonal relaying for security enhancement in untrusted relay networks," in IEEE Int. Conf. Commun. (ICC), Paris, France, May 2017.

[10] L. Musavian and S. Aïssa, "Fundamental capacity limits of cognitive radio in fading environments with imperfect channel information," IEEE Trans. Commun., vol. 57, no. 11, pp. 3472-3480, Nov. 2009.
[11] M. Haghifam, M. R. Mili, B. Makki, M. Nasiri-Kenari, and T. Svensson, "Joint sum rate and error probability optimization: Finite blocklength analysis," IEEE Wireless Commun. Lett., vol. 6, no. 6, pp. 726-729, Dec. 2017.

[12] H. Shariatmadari, S. Iraji, Z. Li, M. A. Uusitalo, and R. Jantti, "Optimized transmission and resource allocation strategies for ultra-reliable communications," in Proc. IEEE Annu. Symp. Personal, Indoor and Mobile Radio Commun. (PIMRC), Valencia, Spain, Sep. 2016.

[13] C. Sun, C. She, and C. Yang, "Energy-efficient resource allocation for ultra-reliable and low-latency communications," in Proc. IEEE Global Commun. Conf. (GLOBECOM), Singapore, Singapore, Dec. 2017.

[14] A. J. Goldsmith, Wireless Communications. Cambridge: Cambridge Univ. Press, 2005.

[15] P. Schniter, "Blind estimation without priors: Performance, convergence, and efficient implementation," Ph.D. dissertation, Cornell University, May 2000.

[16] L. Xiao, Y. Xiao, P. Yang, J. Liu, S. Li, and W. Xiang, "Space-time block coded differential spatial modulation," IEEE Trans. Veh. Technol., vol. 66, no. 10, pp. 8821-8834, Oct. 2017.

[17] L. Musavian, S. Aïssa, and S. Lambotharan, "Adaptive modulation in spectrum-sharing channels under delay quality-of-service constraints," IEEE Trans. Veh. Technol., vol. 60, no. 3, pp. 901-911, Mar. 2011.

[18] C. Li, J. Jiang, W. Chen, T. Ji, and J. Smee, "5G ultra-reliable and low-latency systems design," in Proc. European Conf. Networks and Commun., Oulu, Finland, Jun. 2017.

[19] M. Shehab, E. Dosti, H. Alves, and M. Latva-aho, "On the effective energy efficiency of ultra-reliable networks in the finite blocklength regime," in Proc. IEEE Int. Symp. Wireless Commun. Syst., Bologna, Italy, Aug. 2017.

[20] M. Abramowitz and I. A. Stegun, Handbook of mathematical functions. New York: Dover, 1965.

[21] I. S. Gradshteyn and I. M. Ryzhik, Table of Integrals, Series, and Products. New York: Academic Press, 6th ed., 2000.

[22] M. Geller and E. W. Ng, "A table of integrals of the exponential integral," Journal of Research of the National Bureau of Standards, vol. 73B, no. 3, pp. 191-210, Sep. 1969. 